\title{
Combination chemotherapy consisting of irinotecan, etoposide, and carboplatin (IREC) for refractory or relapsed neuroblastoma
}

\author{
Masayuki Imaya ${ }^{1}$, Hideki Muramatsu ${ }^{1}$, Atsushi Narita ${ }^{1}$, Ayako Yamamori ${ }^{1}$, Manabu \\ Wakamatsu $^{1}$, Taro Yoshida ${ }^{1}$, Shunsuke Miwata ${ }^{1}$, Kotaro Narita ${ }^{1}$, Daisuke Ichikawa ${ }^{1}$, \\ Motoharu Hamada ${ }^{1}$, Eri Nishikawa ${ }^{1}$, Nozomu Kawashima ${ }^{1}$, Nobuhiro Nishio ${ }^{2}$, Seiji \\ Kojima $^{1}$, and Yoshiyuki Takahashi ${ }^{1}$ \\ ${ }^{1}$ Nagoya University Graduate School of Medicine \\ ${ }^{2}$ Nagoya University Hospital
}

December 28, 2020

\begin{abstract}
Background: Patients with relapsed or refractory neuroblastoma have a poor prognosis; there are limited effective and safe rescue chemotherapies for these patients. Development of new chemotherapy regimens for these patients is a key imperative. Procedure: We retrospectively analyzed patients with refractory or relapsed neuroblastoma who received irinotecan, etoposide, and carboplatin (IREC) as a second-line treatment for neuroblastoma. We evaluated the therapeutic response, toxicity, and survival outcomes. We also assessed the impact of UGT1A1 gene polymorphisms, which are involved in irinotecan metabolism, on the outcomes and toxicity. Results: A total of 131 cycles of IREC were administered to 43 patients with a median of two cycles per patient (range, 1-10). All patients were classified as high-risk (International Neuroblastoma Risk Group). Seven patients had relapsed before IREC. One patient $(2 \%)$ showed partial response and 37 patients $(86 \%)$ developed stable disease (disease control rate: 88\%). Grade IV neutropenia was observed in 127 cycles (97\%), while [?] grade III gastrointestinal toxicity was observed in 3 cycles $(2 \%)$. There was no IREC-related mortality. The one-year overall survival and progressionfree survival rates were $65 \%$ and $52 \%$, respectively. Patients with UGT1A1 polymorphisms showed a higher frequency of grade IV neutropenia; however, there was no increase in treatment-related mortality or nonhematological toxicity in these patients. Patients with UGT1A1 gene polymorphisms showed better one-year survival rate than the wild type ( $80 \%$ vs. $44 \%, \mathrm{p}=0.012)$. Conclusions: This study suggests that IREC is well-tolerated by patients with UGT1A1 polymorphisms and is a promising second-line chemotherapy for refractory/relapsed neuroblastoma.
\end{abstract}

\section{Introduction}

Neuroblastoma is the most common extracranial solid tumor in children. The advent of multidisciplinary treatment approach (including chemotherapy, surgery, radiotherapy, and high-dose chemotherapy with autologous peripheral blood stem cell rescue) has helped improve the prognosis of patients with neuroblastoma ${ }^{1}$. However, patients with refractory or relapsed neuroblastoma have unfavorable prognosis ${ }^{2,3}$ despite the development of various salvage therapies, including irinotecan alone $^{4}$, irinotecan with temozolomide ${ }^{5}$, ICE (ifosfamide, carboplatin, and etoposide) ${ }^{6}$, and TOPO-CY (topotecan and cyclophosphamide) ${ }^{7}$ regimens. Therefore, development of new chemotherapy regimens for these patients is a key imperative.

A study reported the outcomes of IRinotecan, Etoposide, and Carboplatin (IREC) in five patients with refractory or relapsed neuroblastoma; one of the patients achieved complete response ${ }^{8}$. No further studies have investigated the outcomes of IREC regimen in a larger cohort. Irinotecan exerts a strong anti-tumor effect by inhibiting the action of type I topoisomerase ${ }^{9}$. Genetic polymorphisms in UGT1A1 are known to be associated with the development of severe side effects of irinotecan, such as diarrhea and leucopenia ${ }^{10}$. 
In this study, we investigated the outcomes of IREC therapy in 43 patients with refractory or relapsed neuroblastoma; in addition, we assessed the impact of UGT1A1 gene polymorphisms on treatment response and toxicity.

\section{Methods}

\section{Patients}

We retrospectively analyzed data pertaining to patients with refractory (defined as inadequate response to treatment that included at least 4 cycles of [?]2 chemotherapeutic agents, including an alkylator and a platinum-containing compound) or relapsed neuroblastoma who underwent IREC therapy at the Nagoya University Hospital between October 2013 and March 2020. Written informed consent for treatment with the IREC regimen was obtained from the patients or their parents prior to the start of chemotherapy. This study was approved by the ethics committee of the Nagoya University Graduate School of Medicine and was conducted in accordance with the principles of the Declaration of Helsinki.

\section{Treatment}

IREC therapy comprised of irinotecan $100 \mathrm{mg} / \mathrm{m}^{2}$ (2-hour infusion) on days 1 through 3 ; etoposide 100 $\mathrm{mg} / \mathrm{m}^{2}$ (2-hour infusion) on days 1 through 3 ; and carboplatin $80 \mathrm{mg} / \mathrm{m}^{2}$ (2-hour infusion) on days 1 through 3. All patients were admitted to the hospital and underwent intravenous hydration. Trimethoprimsulfamethoxazole and fluconazole were administered orally for infection prophylaxis. In patients with grade IV neutropenia, prophylactic use of granulocyte colony-stimulating factor (G-CSF) was allowed. Blood transfusions were administered as appropriate with a target of hemoglobin $>7 \mathrm{~g} / \mathrm{dL}$ and platelet count $>20$ $\times 10^{9} / \mathrm{L}$. Oral cefpodoxime was administered to prevent the development of diarrhea caused by irinotecan. IREC was administered at 4 -week intervals if bone marrow recovery (neutrophil count $>0.5 \times 10^{9} / \mathrm{L}$ and platelet count $>100 \times 10^{9} / \mathrm{L}$ ) was achieved. Treatment response was assessed in all patients using the International Neuroblastoma Response Criteria (INRC) ${ }^{11}$.

\section{Follow-up}

We evaluated the treatment response, survival outcomes, and toxicity using medical records. Follow-up data available as of April 2020 were included in the analysis. Hematological and nonhematological toxicity after IREC therapy were graded according to the National Cancer Institute Common Terminology Criteria for Adverse Events version 5.0.

\section{UGT1A1 genotyping analysis}

UGT1A1 genotyping was performed using the Invader assay, a DNA analysis method that consists of a two-step isothermal reaction using a structure-specific flap endonuclease, as previously reported ${ }^{12}$. Based on the results of testing for $U G T 1 A 1^{*} 6$ and ${ }^{*} 28$ genetic polymorphisms, patients were divided into three groups: wild-type group (-/- ); heterozygous group (*28/- and *6/- ); and homozygous group (*28/*28 , ${ }^{*} 6 /{ }^{*} 6$, and $\left.{ }^{*} 28 /{ }^{*} 6\right)$. Patients with double heterozygosity $\left({ }^{*} 28 /{ }^{*} 6\right)$ were classified into the homozygous group $^{13}$.

\section{Minimal residual disease measurement}

Total RNA was isolated from bone marrow samples using the RNeasy Mini Kit (Qiagen, Valencia, CA); concentrations of the extracted RNA were evaluated by spectrophotometry. The reverse transcription (RT) step was performed using a Thermoscript RT-PCR system (Invitrogen, San Diego, CA) according to the manufacturer's instructions. Real-time quantitative RT-PCR (RQ-PCR) reactions were performed on an ABI Prism 7000 Sequence Detection System (Applied Biosystems, Branchburg, NJ). Ready-made primers and TaqMan probes (Assays-on-Demand Gene Expression Product) for glyceraldehyde-3-phosphate dehydrogenase $(G A P D H)$ Hs99999905_m1, tyrosine hydroxylase $(T H)$ Hs01002188_g1, and paired-like homeobox 2B (PHOX2B ) Hs00243679_m1 were purchased from Applied Biosystems. Each mRNA expression was quantified using TaqMan Universal PCR Master Mix II (Applied Biosystems, Foster City, CA, USA, cat no.4440040). PCR reactions were performed in a total volume of $15 \mu \mathrm{L}$, and the thermal reaction conditions 
were as follows: $50^{\circ} \mathrm{C}$ for $2 \mathrm{~min}, 95^{\circ} \mathrm{C}$ for $10 \mathrm{~min}$, followed by 40 cycles of $95^{\circ} \mathrm{C}$ for $15 \mathrm{sec}, 60^{\circ} \mathrm{C}$ for $1 \mathrm{~min}$, in which fluorescence was acquired and detected by StepOne Real-Time PCR System (Applied Biosystems).

\section{Statistical analysis}

Overall survival (OS) was calculated using the Kaplan-Meier method from the first day of IREC administration to death or the last follow-up visit. The cumulative incidence of relapse and non-relapse mortality was calculated using the Gray test. We used the Chi-squared test to analyze categorical variables and the MannWhitney U test to analyze continuous variables. All statistical analyses were performed using EZR (Saitama Medical Center, Jichi Medical University), which is a graphical user interface for R (The R Foundation for Statistical Computing, Vienna, Austria) ${ }^{14}$.

\section{Results}

\section{Patient Characteristics}

Forty-three patients with refractory or relapsed neuroblastoma had undergone a total of 131 cycles of IREC therapy (Table 1 ). The median age at diagnosis was 3.3 years (range, 1.2-22.7). All patients were classified as high-risk based on the International Neuroblastoma Risk Group classification. At the commencement of IREC therapy, 41 patients (95\%) were $<18$ years of age and the median age (range) was 4.8 (1.8-25.7) years. Twenty patients $(47 \%)$ were male. Eleven patients $(26 \%)$ were refractory to the initial chemotherapies, and seven patients $(16 \%)$ had relapsed before IREC. According to the Lansky ( $<16$ years of age) or Karnofsky performance status scores ([?]16 years of age) at the start of IREC, one patient scored 50, two patients scored 60 and 70 respectively, while the rest of the patients were normally active (score [?]80). All 43 patients had previously been treated with combination chemotherapy, including ICE, TOPO-CY, and irinotecan with TMZ [median cycles of chemotherapy: 10 (range 2-24)]. Before IREC, seven patients (16\%) had received autologous peripheral blood stem cell transplantation, and three (7\%) patients had received cord blood transplantation.

Based on the UGT1A1 genotyping analysis, patients were divided into wild-type group (-/- ) (20 patients), heterozygous group (*28/- and $\left.{ }^{*} 6 /-\right)$ (17 patients), and homozygous group $\left({ }^{*} 28 /{ }^{*} 28,{ }^{*} 6 /{ }^{*} 6\right.$, and ${ }^{*} 28 /{ }^{*} 6$ ) (6 patients).

\section{Treatment Response}

One patient died of tumor progression and tumor bleeding within 6 weeks of initiation of IREC. As the best overall response for the remaining 42 patients, partial response (PR), stable disease, progressive disease (PD) were observed in $1(2 \%), 37(86 \%)$, and $4(9 \%)$ patients, respectively (Table $\mathbf{2}$ ). In one patient (UPN4) who achieved PR, Iodine $123\left({ }^{123} \mathrm{I}\right)$ metaiodobenzylguanidine (MIBG) scintigraphy showed disappearance of MIBG uptake in the bone marrow metastases after the first cycle of IREC, and remained negative until the end of three cycles (Figure 1 ).

In 34 of $43(79 \%)$ patients, bone marrow minimal residual disease (MRD) was measured by quantitative PCR for $T H$ and PHOX2B genes using bone marrow specimens; at baseline (before IREC therapy), 14 patients were MRD positive and 20 were MRD-negative. Five of the 14 patients who were MRD positive at baseline became MRD-negative after IREC therapy. Of the 20 patients who were MRD-negative at baseline, 17 remained negative and 3 patients became MRD positive during IREC therapy.

\section{Toxicity}

A total of 131 cycles (median: 2 cycles/patient; range: 1-10) were administered. Thirty-five cycles required dose reduction for renal damage, prolonged bone marrow suppression, and adjustment of schedule with the subsequent stem cell transplant date. Grade IV hematological toxicity included neutropenia in 127 cycles $(97 \%)$, thrombocytopenia in 38 cycles $(29 \%)$, and anemia in 3 cycles $(2 \%)$. The median duration of grade IV neutropenia was seven days (range: 2-31) with the use of G-CSF support. The median number of transfusions for each cycle was 1 (range: 0-9) for red blood cells and 2 (range: $0-11$ ) for platelets. Of the 43 patients, 35 (81\%) required red blood cell transfusion and $37(86 \%)$ required platelet transfusion. Grade 
IV thrombocytopenia was more common in relapsed cases than in primary cases $(69 \%$ vs $14 \%, p<0.001)$. Nonhematological toxicities of grade III/IV are detailed in Table $\mathbf{3}$. Three patients (2\%) developed grade III diarrhea, while none of the patients developed grade IV diarrhea. Fever of [?]38degC occurred in 58 cycles $(44 \%)$, and febrile neutropenia (FN) occurred in 30 cycles $(23 \%)$. Blood cultures were positive for bacteria in six patients; three of these patients required removal of catheter to control the infection (Table 3 ). Renal and liver damage improved with conservative treatment.

Patients with UGT1A1 gene polymorphism showed a higher frequency of grade IV leukopenia (64\% vs. $94 \%$ ) and neutropenia (94\% vs. 100\%) compared to wild-type patients; however, there was no difference in the frequency of grade III-IV diarrhea or FN.

\section{Survival Outcomes}

The one-year OS rate for the entire cohort was $62 \%$ [95\% confidence interval (CI), 0.427-0.758]. There was no significant difference between primary and relapsed cases with respect to the one-year OS rate (61\% vs. $69 \%, p=0.328)$; patients with PD at IREC initiation showed poorer one-year survival rate compared to the other patients $(21 \%$ vs. $77 \%, p<0.001)$. The cumulative incidence of relapse or PD at one year after IREC was $45 \%$ (95\% CI, 0.279-0.606); patients with PD at the initiation of IREC showed a higher cumulative incidence of relapse or PD compared to the other patients ( $86 \%$ vs. $26 \%, p<0.001$ ).

Patients with $U G T 1 A 1$ gene polymorphism (heterozygous and homozygous groups) showed a better one-year survival rate than wild-type patients ( $77 \%$ vs $48 \%, p=0.026)$ (Figure 2 ); similar results were obtained in an analysis limited to primary cases $(\mathrm{n}=36)$, excluding seven relapsed cases $(80 \%$ vs. $44 \%, p=0.012)$. There was no significant difference in the cumulative incidence of relapse in patients with and without UGT1A1 gene polymorphism (Figure 3 ).

After IREC, 16 patients received a total of 24 stem cell transplantations: 11 autologous peripheral blood stem cell transplantations and 13 cord blood transplantations. Treatment-related death was observed in one patient (UPN15). This patient underwent autologous peripheral blood stem cell transplantation using busulfan + melphalan regimen after three courses of IREC therapy; she died six months after transplantation due to a late-onset non-infectious pulmonary complication.

\section{Discussion}

In this study, we evaluated the efficacy of the IREC therapy in 43 patients with refractory or relapsed neuroblastoma; a high disease control rate of $88 \%$ (38 of 43) was observed in this cohort. This is consistent with the reported in a previous report by Inoue et al. , in which all five patients with refractory or relapsed neuroblastoma achieved disease control with IREC therapy ${ }^{8}$. Although a direct comparison is not appropriate because of the large difference in patient characteristics among the cohorts, the IREC therapy achieved a promising disease control rate, similar to that of other reported salvage therapies for neuroblastoma (ICE, $82 \%^{6}$; TOPO-CY, $62 \%^{7}$; Irinotecan alone, $77 \%^{4}$; and irinotecan + temozolomide, $75 \%^{5}$ ). The IREC regimen would serve as a promising treatment option for patients with refractory or relapsed neuroblastoma.

Grade IV hematological toxicity after IREC treatment was observed in $97 \%$ of patients; however, it was successfully managed with G-CSF and transfusion therapy, and no deaths due to infection were recorded. The main nonhematological toxicity was FN (23\%); other [?] grade III nonhematological toxicities were observed in only $6 \%$ of cases. The incidence of bloodstream infection (BSI) (6 of 43 patients and $6.1 \%$ of all cycles) was lower than that reported with ICE $(26 \%)^{6}$ and was similar to that of irinotecan with temozolomide ( 3 of 47 patients) $)^{5}$. Our results suggest that IREC is a relatively safe chemotherapy regimen for patients with refractory or relapsed neuroblastoma.

Irinotecan is hydrolyzed by carboxylesterase to its active metabolite $\mathrm{SN}-38$, which is then metabolized by UGT1A1 to the non-toxic glucuronide SN-38G. The presence of UGT1A1 gene polymorphisms has been shown to decrease the metabolism of $\mathrm{SN}-38$ and is associated with a higher incidence of neutropenia and diarrhea, which are major side effects of irinotecan treatment ${ }^{9}$. However, the CDE11 regimen including irinotecan for adult diffuse large B cell lymphoma was shown to confer a better prognosis in cases with 
$U G T 1 A 1^{*} 6$ polymorphisms ${ }^{15}$. In the present study, patients with $U G T 1 A 1$ gene polymorphisms showed an increased frequency of grade IV neutropenia, but no increase in FN or BSI, compared to patients with wildtype UGT1A1 . In contrast, patients with the UGT1A1gene polymorphisms showed significantly better 1-year OS and progression-free survival rates than the wild type. Since the toxicity of the IREC regimen was well managed in patients with the gene polymorphisms, future clinical studies should assess whether higher doses of irinotecan can improve the outcomes in patients in the UGT1A1 wild-type group.

In conclusion, this study suggests that IREC is also well-tolerated by patients with UGT1A1 polymorphisms and could be an effective second-line chemotherapy for refractory or relapsed neuroblastoma.

\section{Conflict of Interest statement}

There are no known conflicts of interest.

\section{Acknowledgments}

The authors would like to thank all of the clinicians and families for participating in this study. The authors would like to thank Ms. Yoshie Miura and Ms. Fumiyo Ando for their valuable assistance.

\section{References}

1. Smith MA, Altekruse SF, Adamson PC, Reaman GH, Seibel NL. Declining childhood and adolescent cancer mortality. Cancer . 2014;120(16):2497-2506. doi:10.1002/cncr.28748

2. Kreissman SG, Seeger RC, Matthay KK, et al. Purged versus non-purged peripheral blood stem-cell transplantation for high-risk neuroblastoma (COG A3973): a randomised phase 3 trial. Lancet Oncol . 2013;14(10):999-1008. doi:10.1016/S1470-2045(13)70309-7

3. Pearson AD, Pinkerton CR, Lewis IJ, Imeson J, Ellershaw C, Machin D. High-dose rapid and standard induction chemotherapy for patients aged over 1 year with stage 4 neuroblastoma: a randomised trial. Lancet Oncol . 2008;9(3):247-256. doi:10.1016/S1470-2045(08)70069-X

4. Kushner BH, Kramer K, Modak S, Cheung NK V. Five-day courses of irinotecan as palliative therapy for patients with neuroblastoma.Cancer . 2005;103(4):858-862. doi:10.1002/cncr.20846

5. Kushner BH, Kramer K, Modak S, Cheung NK V. Irinotecan plus temozolomide for relapsed or refractory neuroblastoma. J Clin Oncol . 2006;24(33):5271-5276. doi:10.1200/JCO.2006.06.7272

6. Kushner BH, Modak S, Kramer K, Basu EM, Roberts SS, Cheung NK V. Ifosfamide, carboplatin, and etoposide for neuroblastoma: A high-dose salvage regimen and review of the literature. Cancer . 2013;119(3):665-671. doi:10.1002/cncr.27783

7. Saylors RL, Stine KC, Sullivan J, et al. Cyclophosphamide plus topotecan in children with recurrent or refractory solid tumors: A pediatric oncology group phase II study. J Clin Oncol . 2001;19(15):3463-3469. doi:10.1200/JCO.2001.19.15.3463

8. Inoue M, Yasui M, Sawada A, et al. Efficacy of combination chemotherapy consisting of irinotecan, etoposide, and carboplatin (IREC) in children with refractory solid tumors. Japanese J Pediatr Oncol . 2007;44(2):135-142.

9. Ando Y, Saka H, Ando M, et al. Polymorphisms of UDP-glucuronosyltransferase gene and irinotecan toxicity: A pharmacogenetic analysis. Cancer Res . 2000;60(24):6921-6926.

10. Minami H, Sai K, Saeki M, et al. Irinotecan pharmacokinetics/pharmacodynamics and UGT1A genetic polymorphisms in Japanese: Roles of UGT1A1*6 and *28. Pharmacogenet Genomics . 2007;17(7):497-504. doi:10.1097/FPC.0b013e328014341f

11. Brodeur GM, Pritchard J, Berthold F, et al. Revisions of the international criteria for neuroblastoma diagnosis, staging and response to treatment. Prog Clin Biol Res . 1994;385(8):363-369. doi:10.1080/16501960410016046 
12. Negoro Y, Yano R, Yoshimura M, et al. Influence of UGT1A1 polymorphism on etoposide plus platinuminduced neutropenia in Japanese patients with small-cell lung cancer. Int J Clin Oncol . 2019;24(3):256-261. doi:10.1007/s10147-018-1358-4

13. Satoh T, Ura T, Yamada Y, et al. Genotype-directed, dose-finding study of irinotecan in cancer patients with UGT1A1*28 and/or UGT1A1*6 polymorphisms. Cancer Sci . 2011;102(10):1868-1873. doi:10.1111/j.1349-7006.2011.02030.x

14. Kanda Y. Investigation of the freely available easy-to-use software "EZR" for medical statistics. Bone Marrow Transplant . 2013;48(3):452-458. doi:10.1038/bmt.2012.244

15. Yamasaki S, Tanimoto K, Kohno K, et al. UGT1A1 *6 polymorphism predicts outcome in elderly patients with relapsed or refractory diffuse large B-cell lymphoma treated with carboplatin, dexamethasone, etoposide and irinotecan. Ann Hematol . 2015;94(1):65-69. doi:10.1007/s00277-014-2170-5

\section{Figure Legends}

\section{Figure 1. Metastatic neuroblastoma in an 8-year-old boy}

At the initiation of first IREC therapy, ${ }^{123}$ I-MIBG shows MIBG uptake in the right second rib as a new lesion (A), and continuous uptake in left ileum (B). After three courses of IREC therapy, both lesions are negative for ${ }^{123}$ I-MIBG uptake (C), (D).

IREC, irinotecan, etoposide, and carboplatin; ${ }^{123}$ I-MIBG, Iodine 123 metaiodobenzylguanidine

Figure 2. Overall survival after IREC therapy in patients with relapsed/refractory neuroblastoma

Overall survival (OS) was calculated from the beginning of first IREC therapy to death from any cause. The median duration of follow-up for OS was 9.7 months (range: 0.9-58.3). (A) OS for all patients; (B) OS according to the disease status at the beginning of first IREC therapy; (C) OS according to the UGT1A1 genotype.

IREC, irinotecan, etoposide, and carboplatin.

Figure 3. Cumulative incidence of relapse or progressive disease

Cumulative incidence of relapse or progressive disease (PD) since the initiation of IREC therapy: (A) for all patients; (B) according to the disease status at the beginning of first IREC therapy; (C) according to the UGT1A1 genotype.

IREC, irinotecan, etoposide, and carboplatin.

\section{Hosted file}

Table-1_ver5_MIedit.pdf available at https://authorea.com/users/385646/articles/500912combination-chemotherapy-consisting-of-irinotecan-etoposide-and-carboplatin-irec-forrefractory-or-relapsed-neuroblastoma

\section{Hosted file}

Table-2_ver4_MIedit.pdf available at https://authorea.com/users/385646/articles/500912combination-chemotherapy-consisting-of-irinotecan-etoposide-and-carboplatin-irec-forrefractory-or-relapsed-neuroblastoma

\section{Hosted file}

Table-3_ver4_MIedit.pdf available at https://authorea.com/users/385646/articles/500912combination-chemotherapy-consisting-of-irinotecan-etoposide-and-carboplatin-irec-forrefractory-or-relapsed-neuroblastoma 
Figure 1
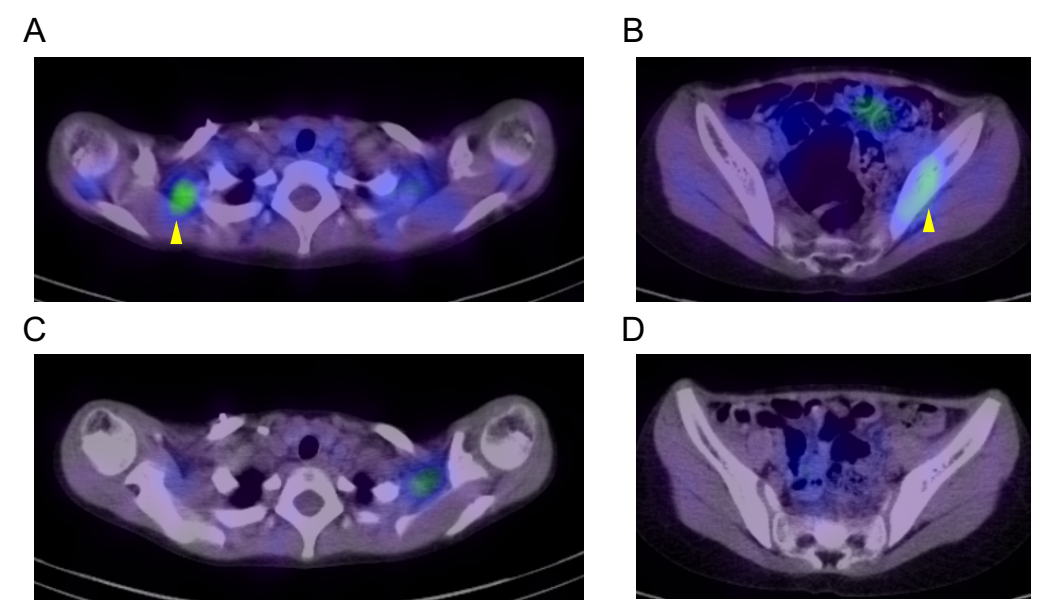


\section{Figure 2}

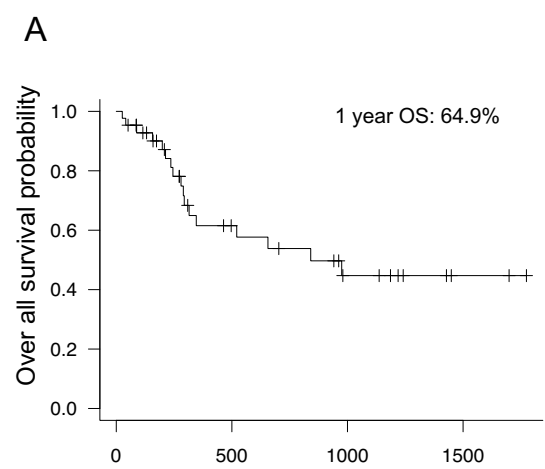

Days from the beginning of first IREC Number at risk

43

16

8

2

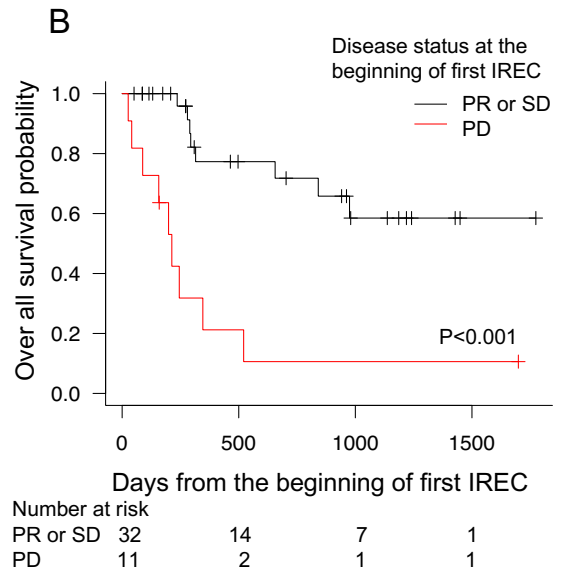

C

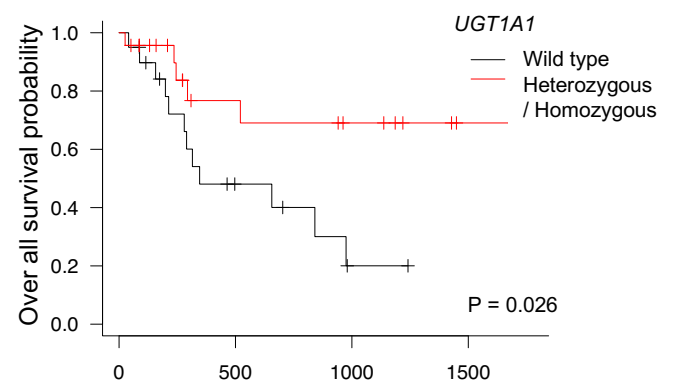

Days from the beginning of first IREC

Number at risk

$\begin{array}{llcll}\text { Wild type } & 20 & 6 & 1 & 0 \\ \text { Hetero } & 23 & 10 & 7\end{array}$

/ Homo 


\section{Figure 3}

A

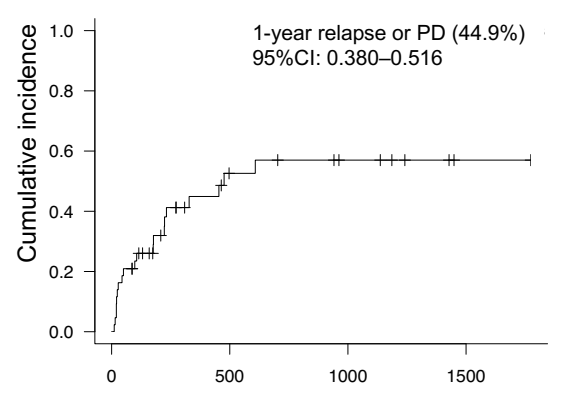

Days from the beginning of first IREC
B

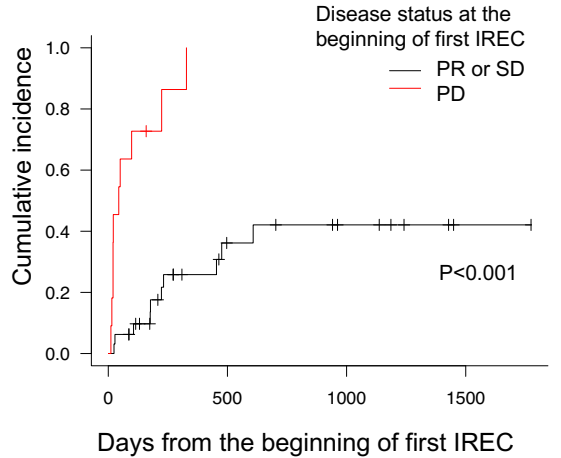

C

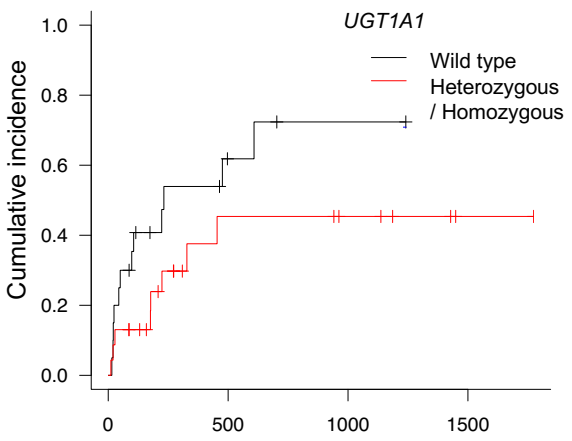

Days from the beginning of first IREC 